\title{
Supplemental Material for \\ Using Score Equating and Measurement Invariance to Examine the Flynn Effect in the Wechsler Adult Intelligence Scale
}

\section{January 2015}

This document provides the syntax for the score equating analyses of the WAIS data. All data analysis was done using the $\mathbf{R}$ statistical programming language (R Development Core Team, 2014), using the equate package (Albano, 2014). For all equating, we created scores to be on the WAIS-III metric. For all subtests, we followed the same procedures, so we only present the syntax for the Similarities subtest for the WAIS-R and WAIS-III equating data. All $\mathbf{R}$ syntax is in a gray box.

\section{Import Data}

The data were stored in two files, one containing WAIS-R and WAIS-III values (WAIS2-3EquatingData.csv), and the other containing WAIS-III and WAIS-IV values (WAIS3-4EquatingData.csv). A picture of the dataset containing WAIS-R and WAIS-III values is given in Figure 1.

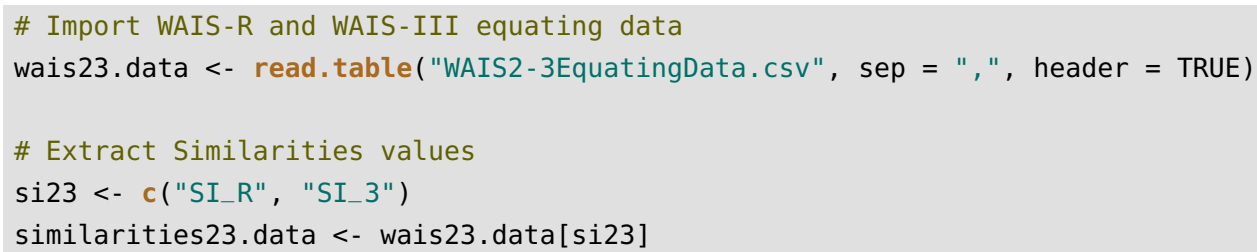

\begin{tabular}{|c|c|c|c|c|c|c|c|}
\hline WAIS3_WAISR_ID & ageyr & ED & ETH & SEX & REGION & SI_R & SI_3 \\
\hline 7 & 17 & 4 & WH & $\mathrm{F}$ & NE & 18 & 20 \\
\hline 24 & 17 & 2 & WH & $\mathrm{F}$ & $\mathrm{NE}$ & 12 & 13 \\
\hline
\end{tabular}

Figure 1. Example values from WAIS-R and WAIS-III equating data.

\section{Descriptive Statistics}

Before equating, we examined the data. A plot of the scores distributions is given in Figure 2. Descriptive statistics are given in Table 1.

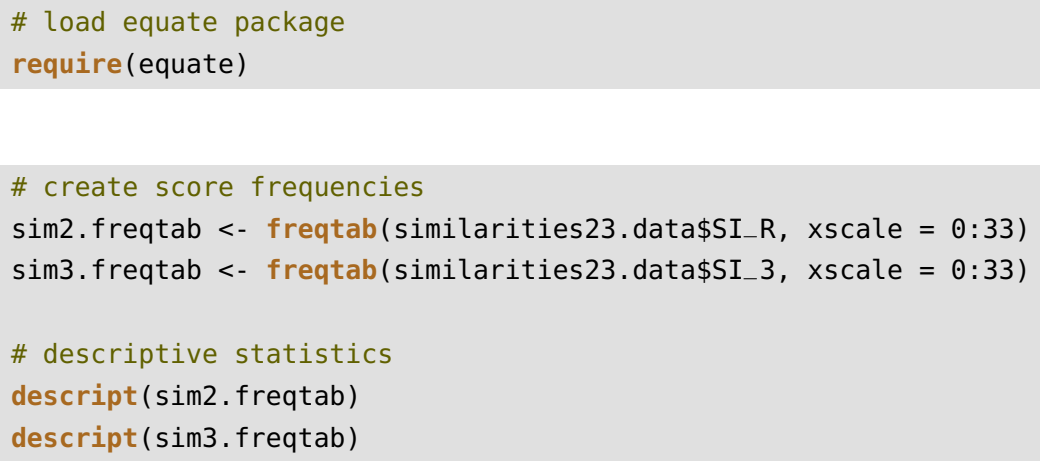




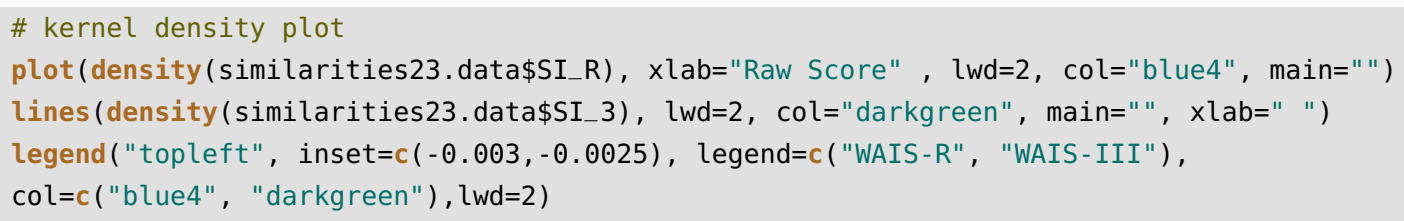

Table 1. Descriptive statistics for the Similarities subtest in WAIS-R and WAIS-III.

\begin{tabular}{rrrrrrrr}
\hline & mean & sd & skew & kurt & $\min$ & $\max$ & $\mathrm{n}$ \\
\hline WAIS-R & 19.62 & 5.02 & -0.99 & 3.86 & 3 & 28 & 192 \\
WAIS-III & 22.34 & 5.35 & -0.74 & 3.29 & 3 & 32 & 192 \\
\hline
\end{tabular}

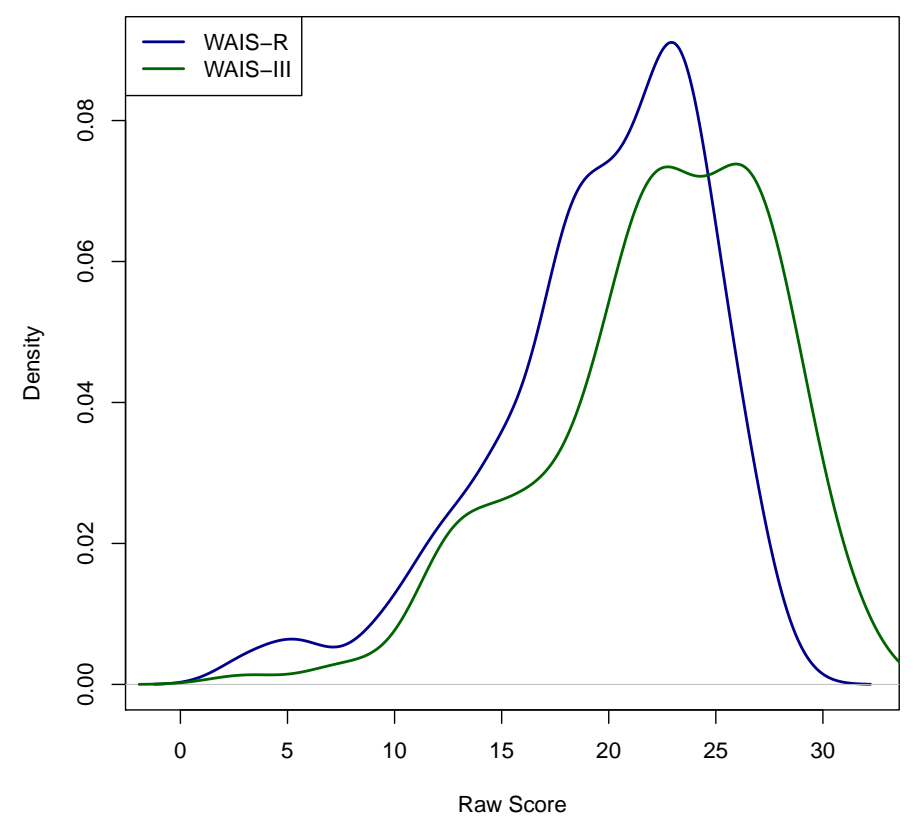

Figure 2. Kernel density plots of the WAIS-R and WAIS-III Similarities values from equating data.

\section{Pre-smoothing}

We examined different powers for the pre-smoothing. The pre-smoothing results for the WAIS-R are given in Table 2 and the results for the WAIS-III are given in Table 3. For both the WAIS-R and the WAIS-III, a power of 4 seems to work the best; thus, a quartic model was used for the equating. Graphs of the original and pre-smoothed distributions are given in Figure 3.

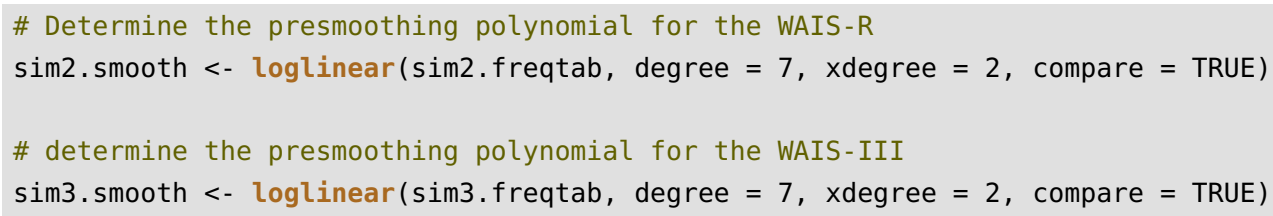


Table 2. Results from different polynomials of polynomial log-linear pre-smoothing for the WAIS-R Similarities subtest. First column contains the highest power of the polynomial.

\begin{tabular}{rrrr}
\hline & df & Deviance & AIC \\
\hline 1 & 32 & 264 & 350 \\
2 & 31 & 92 & 180 \\
3 & 30 & 63 & 153 \\
4 & 29 & 53 & 145 \\
5 & 28 & 52 & 146 \\
6 & 27 & 48 & 144 \\
7 & 26 & 47 & 146 \\
\hline
\end{tabular}

Table 3. Results from different polynomials of polynomial log-linear pre-smoothing for the WAIS-III Similarities subtest. First column contains the highest power of the polynomial.

\begin{tabular}{rrrr}
\hline & df & Deviance & AIC \\
\hline 1 & 32 & 171 & 263 \\
2 & 31 & 67 & 160 \\
3 & 30 & 57 & 153 \\
4 & 29 & 51 & 148 \\
5 & 28 & 49 & 149 \\
6 & 27 & 49 & 151 \\
7 & 26 & 49 & 153 \\
\hline
\end{tabular}

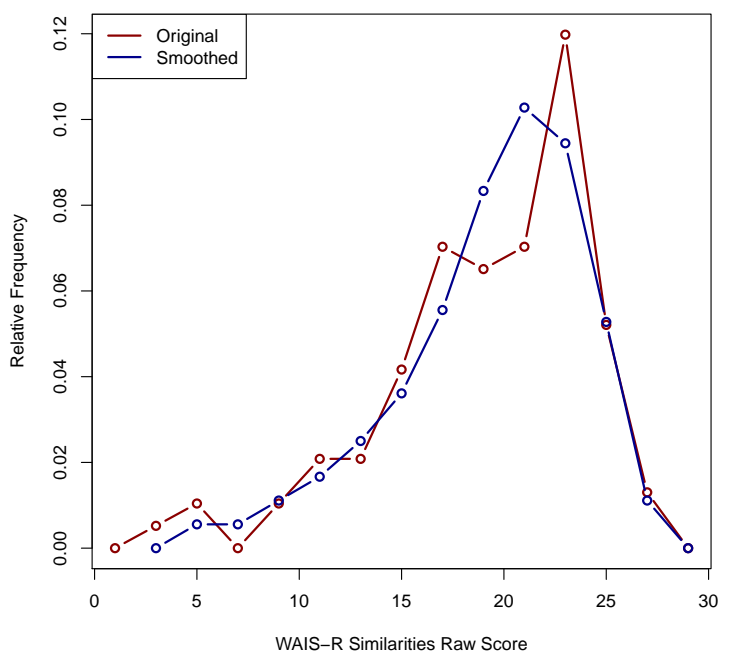

(a) WAIS-R Similarities distribution (log linear $C=4)$.

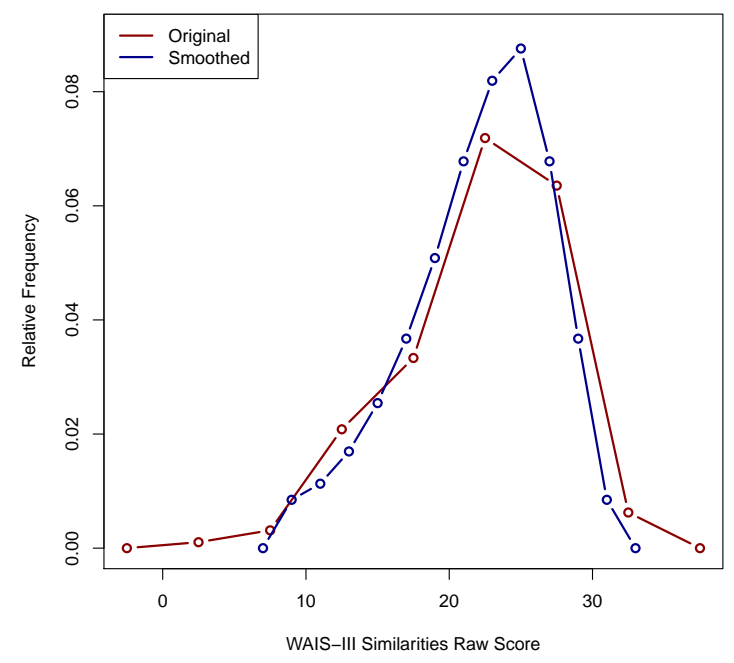

(b) WAIS-III Similarities distribution (log linear $C=4)$.

Figure 3. Presmoothing WAIS Similarities distributions. 


\section{Equating}

We equated the subtest scores using equipercentile equating with smoothing. In addition, we calculated the bootstrapped standard error of equating (SEE). A figure of the SEE by Similarities score is given in Figure 4. A concordance table based on Equipercentile equating with smoothing equating is given in Table 4.
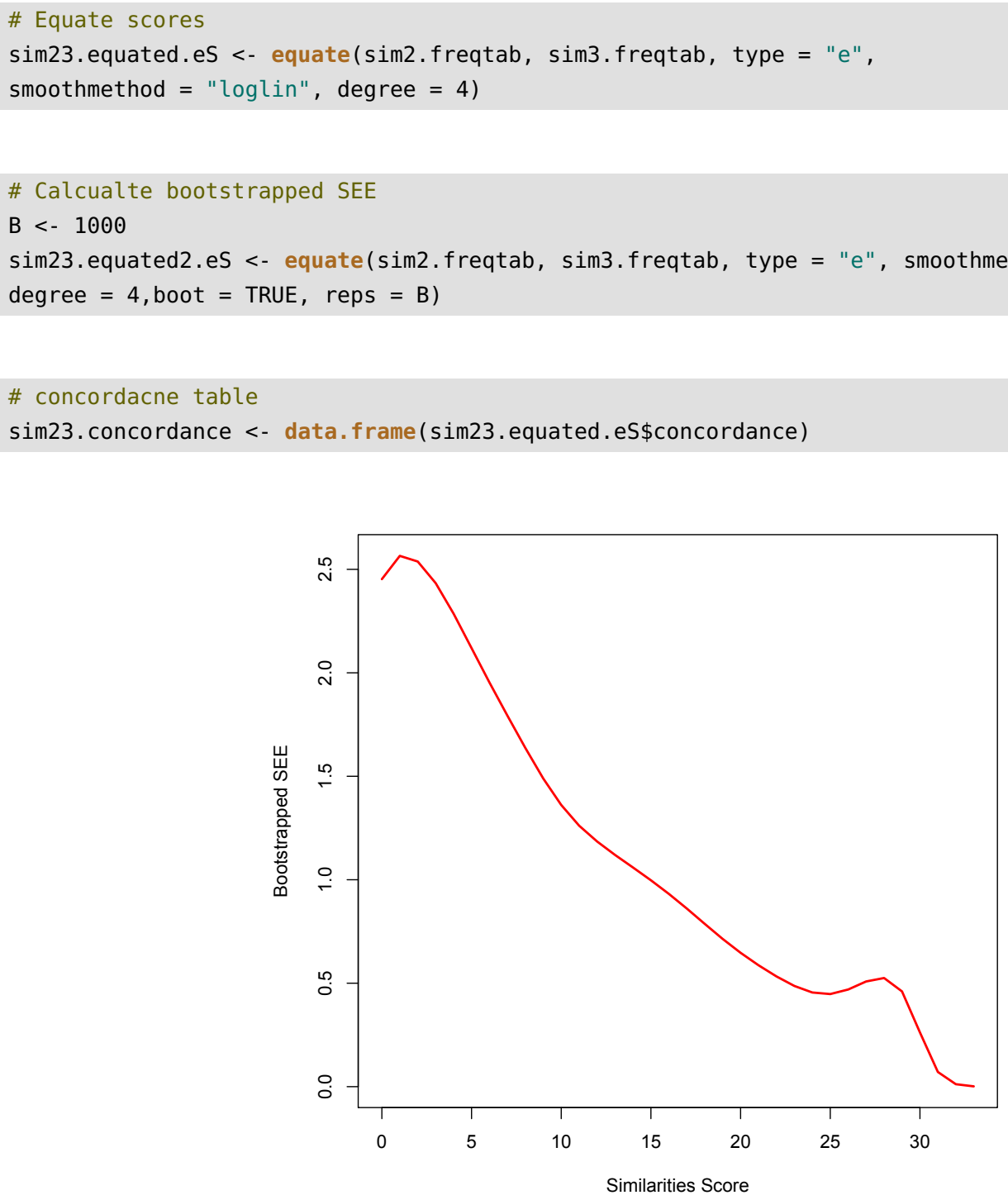

Figure 4. Bootstrapped standard error of equating for the WAIS-R Similarities subtest (B=1000). 
Table 4. Sample Values from Concordance Table for the WAIS-R Similarities Subtest. Values are rounded to the nearest integer.

\begin{tabular}{ll}
\hline Original & Equated \\
\hline 0 & 2 \\
1 & 4 \\
2 & 5 \\
3 & 6 \\
4 & 7 \\
$\cdot$ & $\cdot$ \\
$\cdot$ & $\cdot$ \\
. & $\cdot$ \\
29 & 33 \\
30 & 33 \\
31 & 33 \\
32 & 33 \\
33 & 33 \\
\hline
\end{tabular}




\section{References}

Albano, A. (2014). equate: Observed-score linking and equating [Computer software]. Retrieved from http : //CRAN. R-project . org/package=equate.

R Development Core Team. (2014). R: A language and environment for statistical computing [Computer program]. Vienna, Austria: R Foundation for Statistical Computing. 